\title{
A comparison of multiple shRNA expression methods for combinatorial RNAi
}

\author{
Glen J Mcintyre ${ }^{1,2^{*}}$, Allison J Arndt', Kirsten M Gillespie', Wendy M Mak ${ }^{1}$ and Gregory C Fanning ${ }^{1,3}$
}

\begin{abstract}
RNAi gene therapies for HIV-1 will likely need to employ multiple shRNAs to counter resistant strains. We evaluated 3 shRNA co-expression methods to determine their suitability for present use; multiple expression vectors, multiple expression cassettes and single transcripts comprised of several dsRNA units (aka domains) with each being designed to a different target. Though the multiple vector strategy was effective with 2 shRNAs, the increasing number of vectors required is a major shortcoming. With single transcript configurations we only saw adequate activity from 1 of 10 variants tested, the variants being comprised of $2-3$ different target domains. Whilst single transcript configurations have the most advantages on paper, these configurations can not yet be rapidly and reliably re-configured for new targets. However, our multiple cassette combinations of 2, 3 and 4 (29 bp) shRNAs were all successful, with suitable activity maintained in all positions and net activities comparable to that of the corresponding single shRNAs. We conclude that the multiple cassette strategy is the most suitably developed for present use as it is easy to design, assemble, is directly compatible with pre-existing shRNA and can be easily expanded.
\end{abstract}

\section{Introduction}

The recently discovered RNA interference (RNAi) pathway is a post-transcriptional gene silencing and regulation mechanism with potential application in the field of gene therapy. In mammalian cells RNAi begins with a double-stranded RNA inducer that is progressively processed from its termini by RNase III type endonucleases, firstly Drosha in the nucleus followed by Dicer in the cytoplasm, to yield a short interfering RNA (siRNA) duplex of $\sim 22$ bp [1,2]. The duplex is unwound and loaded into the RNA induced silencing complex (RISC) in a process that favors one of the two strands (the guide strand) based on a difference in thermodynamic stability at the ends of the duplex [3]. The most common natural substrates for mammalian RNAi are microRNA, short hairpin-like RNA transcripts implicated in regulating gene expression activity $[1,2]$. The RNAi pathway can be artificially engaged at any point in the process, typically either through delivering synthetic siRNAs to the RISC $[4,5]$ or by expressing short hairpin RNAs (shRNA or

\footnotetext{
* Correspondence: glen@madebyglen.com

'Johnson and Johnson Research Pty Ltd, Level 4 Biomedical Building,

1 Central Avenue, Australian Technology Park, Eveleigh, NSW, 1430, Australia Full list of author information is available at the end of the article
}

hairpins) to be processed by Dicer and possibly Drosha $[6,7]$.

shRNAs are well suited for use in current gene therapy plans. shRNA consists of a short single-stranded RNA transcript that folds into a 'hairpin' configuration by virtue of self-complementary regions separated by a short 'loop' sequence. Whilst hairpins can be expressed from either polymerase (pol) III or more recently pol II promoters, it is the U6 and $\mathrm{H} 1$ pol III promoters that have been most extensively employed owing in part to their relatively welldefined transcription start and end points [6,7]. Importantly, pol III based hairpin expression cassettes have been incorporated into viral vectors which have been stably integrated both in culture and whole animals with effective silencing maintained over time [8-10]. The potency of individual shRNA directed to HIV has been extensively demonstrated [11-13], however, several studies have also shown single shRNA can be rapidly overcome by the emergence of escape mutants [14-17]. Modeling shows that perhaps as few as 4 shRNAs used in combination may be sufficient to prevent the emergence of escape mutants [18-22]. This idea is supported by several wet studies showing that in laboratory conditions HIV-1 escape can be delayed by using more than one shRNA [11,23-26]. In a clever variation on this idea, some have designed

\section{() Biomed Central}


anticipatory shRNAs specifically to block known escape routes, though when tested it was found that the virus still evolved around these $[27,28]$. There is now clearly a need for an evaluation of multiple shRNA expression strategies to identify those that can be readily integrated into current anti-HIV gene therapy research programs.

One method for expressing multiple shRNAs is to use separate expression vectors encoding individual shRNA. Multiple shRNAs have been used successfully against cellular, viral and exogenous gene targets by the use of either multiple plasmid [29], retrovirus [30] or lentivirus vectors [9]. Multiple shRNAs can also be combined into a single expression vector via several self-contained expression cassettes (e.g. 1 cassette = promoter, shRNA and terminator), of which there are now many examples [11,31-34]. Alternatively, multiple shRNA domains can be combined in a single transcript, of which there are two base configurations; distinct hairpin domains joined 3 ' to 5' in what we call a 'cluster' (CL) configuration, and a 'head-to-tail' (HT) configuration in which all the sense stem regions are joined first, followed by a single loop and then all the anti-sense regions [17,35-39]. This second configuration appears as a long hairpin which, depending on the design, may be punctuated by unpaired spacer regions between the hairpin domains. Single transcript strategies are the most compact, and in this respect the most desirable means of co-expressing multiple shRNAs for gene therapy, but with few examples and no design guidelines yet reported, its general ease-of-use is unclear.

The aim of this study was to evaluate the 3 different shRNA co-expression methods to determine their suitability for present use in gene therapy schemes, with a key focus on ease of construction, applicability to new sequences, and the retention of suppressive activity in the component shRNAs. We assembled combinations of 2 to 4 shRNAs using 3 different strategies: one using multiple expression vectors, one using multiple hairpin expression cassettes and the other based on a single transcript comprised of different hairpin domains. While we were able to achieve successful suppression with each strategy, we concluded that the multiple cassette strategy is currently most useful due to ease of design, assembly, and its immediate compatibility with preexisting shRNA already selected for high activity.

\section{Results}

\section{Co-expression using multiple vectors}

The effects of co-expressing hairpins of different sequence were first examined in its simplest form; using two hairpins, each expressed from a separate plasmid vector (pS) derived from pSilencer 3.0-H1 (Ambion). In this circumstance the suppressive activities of each hairpin were measured when separately expressed, and then again when co-expressed. All hairpins in this study were expressed from the human $\mathrm{H} 1$ polymerase III promoter. This experiment employed two 29 bp shRNAs against the HIV-1 genes encoding for Tat and Vif: Tat 56-29 (T) and Vif 88-29 (V) (variant NL4-3, accession \#AF324493) (Table 1). Suppressive activity was measured by flow cytometry as a reduction in fluorescence of an appropriate reporter after transfection and transient expression of both hairpin(s) and reporter(s) in HEK293a cells. In this and all subsequent experiments the activity of each shRNA vector was measured relative to the activity of the appropriate control vector containing an equivalent number of 'empty' (e) expression cassettes (i.e. consisting of a promoter(s) plus terminator but expressing no hairpin(s)).

Either the two hairpin vectors, or each hairpin vector and the equivalent control vector were co-transfected at different ratios (Figure 1). The total amount of DNA delivered for each transfection was kept constant and the shRNA expression vector was always present at a level such that the RNAi process would presumably be saturated so that the effects of competition, if any, would be evident [40]. As each hairpin was directed to a different target the suppressive activity was measured using two unique reporters (GFPsTat and AsRed1sVif), which could be detected both simultaneously and independently. The specific activity of each hairpin vector was unaffected in the presence of empty expression vector at all ratios. However, the specific activity of each hairpin when co-expressed was progressively reduced at ratios that increasingly favored the competing hairpin. We surmise that reduction in specific activity was not due to a second vector (or promoter), but rather from inter-hairpin competition for access to the RNAi machinery. In summary, co-expressed hairpins delivered via separate vectors can function simultaneously, but do so at reduced levels due to competitive access for the RNAi machinery.

\section{Co-expression using multiple-cassette vectors: establishing positional effects}

An alternative strategy to overcome the obvious limitations of using multiple vectors to co-express hairpins (e.g. issues of vector multiplicity) was to incorporate multiple hairpin expression cassettes within a single vector. However, to address concerns of potential promoter or transcriptional interference [41] it was important to first determine if each individual cassette position in a multiple cassette vector was capable of expressing a hairpin that functioned with equivalent suppressive activity. At this point we switched to using a pLenti6 derived vector backbone ( $\mathbf{p L}$ ) (Invitrogen) so that we could later test our constructs in stably transduced scenarios. We assembled a series of 1, 2, 3 and 4 cassette vectors containing only a single hairpin $(\mathbf{T})$ expression cassette per vector, which 
Table 1 shRNA sequences

\begin{tabular}{|c|c|c|c|c|c|c|}
\hline $\mathrm{r}^{\mathrm{a}}$ & Target & $\ln ^{\mathrm{b}}$ & Stem (sense) & Loop & Te. $^{\mathbf{b}}$ & Len. \\
\hline $\mathbf{T}$ & Tat 56-29 & G & AAACUGCUUGUACCAAUUGCUAUUGUAAA & ACTCGAGA & G & 70 \\
\hline v & Vif $88-29$ & G & UAUAUUUCAAGGAAAGCUAAGGACUGGUU & UCTCGAGU & - & 69 \\
\hline $\mathbf{R}$ & Vpr $72-29$ & - & GGAACUUAAGAGUGAAGCUGUUAGACAUU & ACTCGAGA & - & 68 \\
\hline U & Vpu 158-29 & - & GCAAUGAGAGUGAAGGAGAAGUAUCAGCA & ACTCGAGA & - & 68 \\
\hline $\mathbf{O}^{\prime}$ & Off \#1-29 & - & AAGACAGUCCAACACACGCCACCUGUCUC & UCTCGAGU & - & 68 \\
\hline $\mathrm{O}^{\prime \prime}$ & Off \#2-29 & - & AACAGUCUGUCAAAGGUGACCCCUGUCUC & UCTCGAGU & - & 68 \\
\hline $\mathrm{T}^{19}$ & Tat 59-19 & - & CUGCUUGUACCAAUUGCUA & UUCUCGAGA & - & 50 \\
\hline$V^{19}$ & Vif 98-19 & - & GGAAAGCUAAGGACUGGUU & UCTCGAGU & - & 48 \\
\hline
\end{tabular}

a: shRNA abbreviation.

b: In. (Initiating)/Te. (Terminating): Extra nucleotides included 5'(the first nt. transcribed) and $3^{\prime}$ (the last nt. transcribed prior to 1 or more $\mathrm{U}$ termination remnants) in only some sequences (those which were also a part of an overlapping study).

was placed at each different position. The surrounding positions consisted of 'empty' expression cassettes, e.g. the 3 cassette vectors included 3.1: $\mathbf{T}+\mathbf{e}+\mathbf{e}, 3.2: \mathbf{e}+\mathbf{T}+\mathbf{e}$, and 3.3: $\mathbf{e}+\mathbf{e}+\mathbf{T}$. There was $\sim 130 \mathrm{bp}$ of spacer sequence between each cassette, as measured from the terminator of one cassette (n) to the promoter of the next downstream cassette $(n+1)$. Control vectors were also created that were of corresponding sizes and were composed of an equivalent number of all empty expression cassettes ( 2 to 4). Suppressive activity was first measured by flow cytometry as a reduction in fluorescence after transfection and transient expression from both hairpin and reporter vectors (Figure 2a). There was no apparent reduction in activity from any cassette position, from either the 2,3 or 4 cassette vectors, with each cassette position retaining full hairpin activity equivalent to the single position, single hairpin cassette vector.

\section{Co-expression using multiple-cassette vectors: multiplication of a single hairpin}

pLenti6 based vectors with 2,3 , and 4 cassettes were constructed with an identical hairpin (T) expression cassette placed in all positions to investigate whether increasing the cassette number could increase the suppressive activity (e.g. $2 \times: \mathbf{T}+\mathbf{T}, 3 \times: \mathbf{T}+\mathbf{T}+\mathbf{T}$, and $4 \times: \mathbf{T}+\mathbf{T}+\mathbf{T}+\mathbf{T}$ ). Suppressive activity was measured across a range of vector amounts (from $400-0 \mathrm{ng}$ ) so that the effects of increasing cassette number could be investigated during both RNAi-saturating and sub-saturating conditions (Figure 2b). The total amount of DNA delivered for each transfection was kept constant by supplementing each reaction with the appropriate amount of corresponding control vector whilst keeping the amount of reporter vector constant. There were no differences in the suppressive activities from $400 \sim 100 \mathrm{ng}$ of each vector delivered, which supported the hypothesis that the RNAi process was saturated across this range. However, at $50-10 \mathrm{ng}$ of vector(s) there were statistically significant improvements in suppressive activity $(*)$ with increasing cassette numbers $(\mathrm{P}<0.05$, comparing the single cassette vector to the 2,3 , and 4 cassette vectors). The trend did not extend below this concentration range, as effective suppressive activity was lost and accordingly any meaningful difference between the different numbers of cassettes.

We further examined whether multiplying an identical expression cassette would be beneficial in stably transduced cell lines. Infectious virus was generated from each of the 1, 2, 3 and 4 (T) cassette vectors, along with the

\section{(a)}

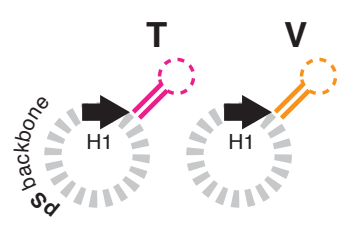

(b)

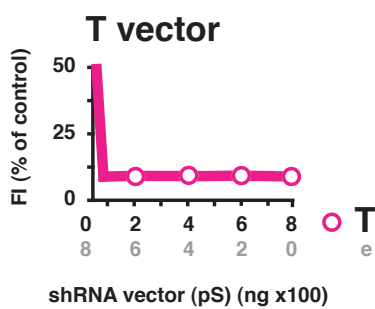

(c)

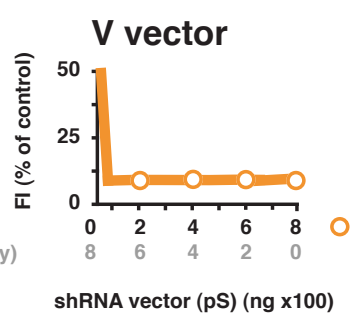

(d)

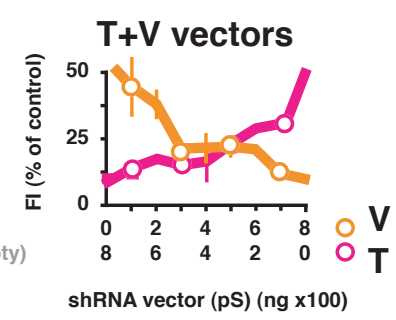

Figure 1 Activities of different shRNAs from different vectors. (a) The first two test hairpins, Tat 56-29 (T) and Vif $88-29$ (V), were expressed from separate plasmid vectors (pS) via the H1 polymerase III promoter. HEK293a cells were transfected with 800 ng of the pSilencer-based (pS) $T$ vector (+ empty) (b), the $V$ vector (+ empty) (c), or the $T$ and $V$ vectors together (d) at mass ratios of 1:7 to 7:1, plus 100 ng each of both target-specific reporter vectors GFPsTat (T.r) and AsRed1sVif (V.r-red). There were 9 data points plotted for each curve, though for clarity open circles are only shown on every 2 nd one. The data shown is representative of several replicated experiments (repeated twice, i.e. $n=2$ ). 


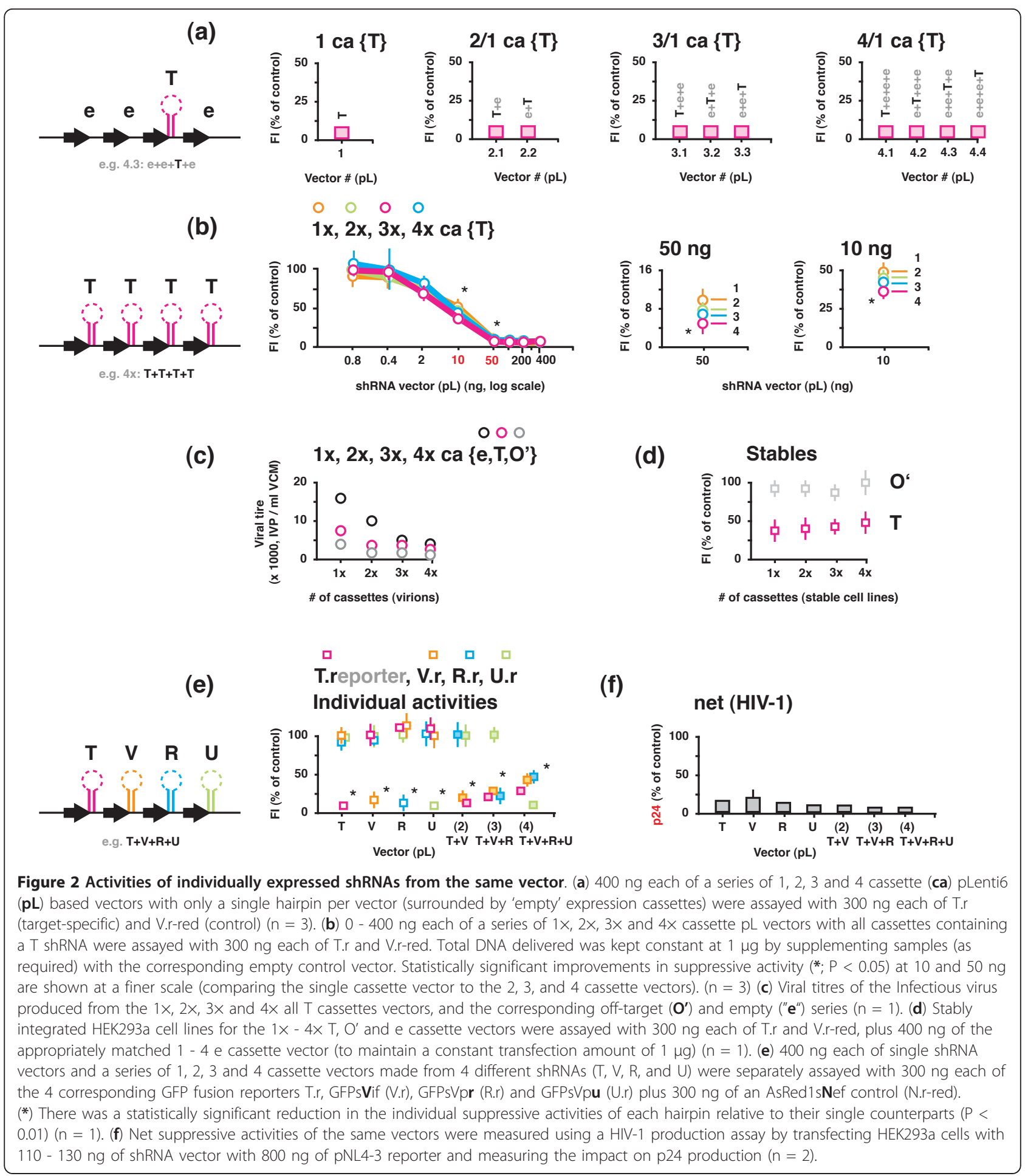

empty control vectors, and another corresponding series of off-target shRNA cassette vectors (using an unmatched, off-target, 29 bp hairpin $\left(\mathbf{O}^{\prime}\right)$ in 1, 2, 3, and 4 cassette combinations). Interestingly, when preparing virus, we saw a steady decrease in titre that correlated with increasing cassette number for both hairpin containing and empty expression cassette vectors (Figure 2c). We infected (transduced) HEK293a cells, selected for stable integrants, and measured suppressive activity by transfecting and transiently expressing the relevant reporter vectors into each cell line (Figure 2d). Analysis of the off-target hairpin controls showed no deleterious impact on fluorescence 
activity from the vector backbone, promoters or irrelevant hairpins. However, the suppression levels from the Tatspecific shRNA cell lines were reduced by $\sim 30-40 \%$ relative to the maximum levels observed during transient expression (cf. Figure 2b), which was not unexpected and most likely due to low-copy number integration with a corresponding reduction in shRNA expression $[8,18,42]$. In this stable expression system there were no statistically significant differences in suppressive activity between the 2, 3 and 4 cassette cell lines.

\section{Co-expression using multiple-cassette vectors: diversifying hairpin targets}

The multiple cassette strategy was further investigated by combining four different shRNA in a single vector using the same $\mathrm{pL}$ base vectors and cassette configurations as before. In addition to Tat 56-29 and Vif 88-29, two further (HIV-1) 29 bp shRNA were used: Vpr 72-29 (R) and Vpu 158-29 (U). Each shRNA was placed in both single cassette vectors and combination vectors of $2(\mathbf{T}+\mathbf{V}), 3(\mathbf{T}+\mathbf{V}+\mathbf{R})$ and $4(\mathbf{T}+\mathbf{V}+\mathbf{R}+\mathbf{U})$ cassettes. All vectors were assayed in turn with each of the relevant reporter vectors enabling the specific activity of each shRNA to be measured independently (Figure 2e). Each individual hairpin exhibited potent and specific activity for its matched assay vector(s). There was, however, a progressive and statistically significant reduction in the individual suppressive activity of each hairpin when expressed in combinations of increasing number, relative to the corresponding individual shRNA $(\mathrm{P}<0.01)$, the exception being Vpu 158-29, which appeared unaffected. The activity of each vector was further examined using HIV-1 as the target and p24 production (a capsid protein) as the readout (Figure 2f). Whilst each hairpin was of a different sequence, their activities were now measured via a common endpoint (p24 production) and thus each could be considered as being directed towards a "common or shared target". The net suppressive activity of each multiple hairpin vector was similar to the corresponding individual hairpin vectors (all within 5\% of each other). We concluded that whilst hairpins compete with each other for access to RNAi machinery, a net suppressive activity can be maintained from multiple simultaneously acting shRNAs.

\section{Co-expression using single transcript arrays of hairpin domains}

The last co-expression strategy tested was the single transcript strategy comprised of several shRNA domains (or technically just dsRNA domains, depending on design). There are two basic configurations, the 'cluster' (CL) configuration and the 'head-to-tail' (HT) configuration. Using pSilencer based vectors, we constructed two cluster arrays of three 29 bp hairpin domains $\left(\mathbf{R}_{-} \mathbf{V}_{-} \mathbf{T}_{\mathbf{1}}\right.$ and $\mathbf{R}_{-} \mathbf{V}_{-} \mathbf{T}_{\mathbf{8}}$ ) with either 1 or 8 nt. spacers separating each domain (Table 2), and tested them with our fluorescent reporters (Figure 3a). Despite reported success from others $[43,44]$, we only saw good activity in the first domain, with poor activity in the remaining two.

Following this we created a head-to-tail configuration comprised of two 29 bp hairpin domains $(\mathbf{V}-\mathbf{T})$ separated by an $8 \mathrm{nt}$. spacer. Suppressive activity was measured for both domains simultaneously by using dual reporters (GFPsTat and AsRed1sVif), which showed that each domain was simultaneously active (Figure $3 \mathrm{~b}$ ). We also show that this result was target-specific, since a second control molecule similarly constructed from two 29 bp offtarget hairpin domains (O'-O') showed no suppressive activity (Figure 3c). We further transferred the V-T headto-tail configuration into a our pLenti6 based plasmid and assayed it using HIV-1 (i.e. a shared target) (Figure 3d). The net suppressive activity was comparable to the activities of the corresponding individual shRNAs and the combined activities of both individual shRNAs when delivered via the equivalent multiple cassette strategy. Encouraged by this finding, we assembled and tested several more headto-tail configurations, comprised of the same domains but in different order (T-V), different domains (U-R and $\mathbf{R}-\mathbf{U})$, more domains ( $\mathbf{R}-\mathbf{V}-\mathbf{T}$ and $\mathbf{T}-\mathbf{V}-\mathbf{R})$, no spacers $\left(\mathbf{V}-\mathbf{T}_{\mathbf{o}}\right)$, and shorter 19 bp domains $\left(\mathbf{V}^{\mathbf{1 9}}-\mathbf{T}^{\mathbf{1 9}}\right)$. However, in no case did we achieve a similarly successful outcome to our original V-T molecule (Figure 3e-h). While some domains were active, others were not, and no configuration retained comparable activity to the corresponding component shRNAs for all domains. We conclude that while two hairpins combined in a head-to-tail configuration can be successful, reliably obtaining an active molecule requires a more detailed design (than simply connecting pre-existing hairpins) that will come from a better understanding of how these configurations are processed.

\section{Discussion}

In this study we tested 3 different strategies for the simultaneous expression of multiple hairpins and showed that all could be effective. But, the multiple vector strategy is likely to be of limited use in gene therapy since it requires a unique vector per shRNA, with potential issues in ensuring that each cell receives all vectors (without which may facilitate the emergence of resistant strains). The single transcript strategy was effective in one instance, but since similar success was not reproduced with different domains (or configurations), it is also of limited use in its present form. However, the multiple expression cassette strategy was used successfully with up to 4 shRNAs, and was easy to assemble and expand with pre-selected shRNAs.

When hairpins were co-expressed at levels that saturated the RNAi process we found that the individual 
Table 2 Multiple domain, single transcript sequences

\begin{tabular}{|c|c|c|}
\hline Comb. & Sequence $^{a}$ & Len. \\
\hline$\overline{R \_V_{-} T_{1}}$ & 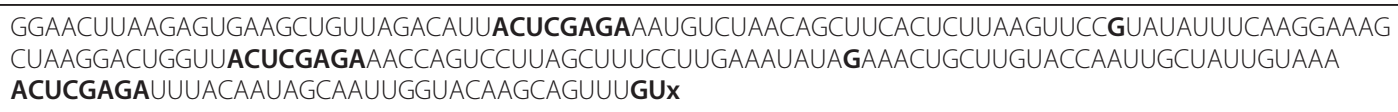 & 204 \\
\hline R_V_T 8 & 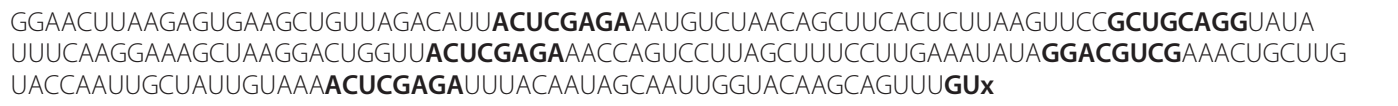 & 218 \\
\hline V-T & $\begin{array}{l}\text { GUAUAUUUCAAGGAAAGCUAAGGACUGGUUGCUGCAGGAAACUGCUUGUACCAAUUGCUAUUGUAAAACUCGAGA } \\
\text { UUUACAAUAGCAAUUGGUACAAGCAGUUUGGACGUCGAACCAGUCCUUAGCUUUCCUUGAAAUAUAUx }\end{array}$ & 144 \\
\hline $\mathrm{O}^{\prime}-\mathrm{O}^{\prime \prime}$ & $\begin{array}{l}\text { AAGACAGUCCAACACACGCCACCUGUCUCGCUGCAGGAACAGUCUGUCAAA } \\
\text { GGUGACCCCUGUCUCACUCGAGAGAGACAGGGGUCACCUUUGACAGACUGUUGGACGUCGGAGACAGGUGGCGUGU } \\
\text { GUUGGACUGUCUUGUx }\end{array}$ & 144 \\
\hline T-V & $\begin{array}{l}\text { GAAACUGCUUGUACCAAUUGCUAUUGUAAAGCUGCAGGUAUAUUUCAAGGAAAGCUAAGGACUGGUUACUCGAGA } \\
\text { AACCAGUCCUUAGCUUUCCUUGAAAUAUAGGACGUCGUUUACAAUAGCAAUUGGUACAAGCAGUUUGUx }\end{array}$ & 145 \\
\hline U-R & $\begin{array}{l}\text { GGCAAUGAGAGUGAAGGAGAAGUAUCAGCAGCUGCAGGGGAACUUAAGAGUGAAGCUGUUAGACAUU } \\
\text { ACUCGAGAAAUGUCUAACAGCUUCACUCUUAAGUUCCGGACGUCGUGCUGAUACUUCUCCUUCACUCUCAUUGCGUx }\end{array}$ & 145 \\
\hline$R-U$ & $\begin{array}{l}\text { GGGAACUUAAGAGUGAAGCUGUUAGACAUUGCUGCAGGGCAAUGAGAGUGAAGGAG } \\
\text { AAGUAUCAGCAACUCGAGAUGCUGAUACUUCUCCUUCACUCUCAUUGCGGACGUCGAAUGUCUAACAGCUUCACU } \\
\text { CUUAAGUUCCGUx }\end{array}$ & 145 \\
\hline R-V-T & 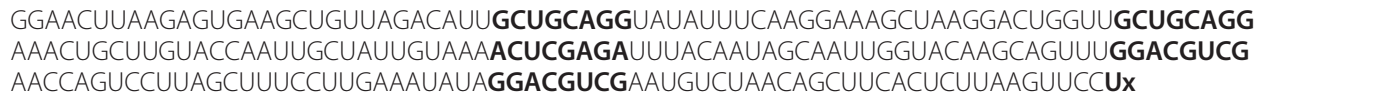 & 217 \\
\hline T-V-R & $\begin{array}{l}\text { AAACUGCUUGUACCAAUUGCUAUUGUAAAGCUGCAGGUAUAUUUCAAGGAAAGCUAAGGACUGGUUGCUGCAGG } \\
\text { GGAACUUAAGAGUGAAGCUGUUAGACAUUACUCGAGAAAUGUCUAACAGCUUCACUCUUAAGUUCCGGACGUCG } \\
\text { AACCAGUCCUUAGCUUUCCUUGAAAUAUAGGACGUCGUUUACAAUAGCAAUUGGUACAAGCAGUUUUx }\end{array}$ & 217 \\
\hline $\mathrm{V}-\mathrm{T}_{0}$ & $\begin{array}{l}\text { GUAUAUUUCAAGGAAAGCUAAGGACUGGUUAAACUGCUUGUACCAAUUGCUAUUGUAAAACUCGAGA } \\
\text { UUUACAAUAGCAAUUGGUACAAGCAGUUUAACCAGUCCUUAGCUUUCCUUGAAAUAUAUx }\end{array}$ & 128 \\
\hline$V^{19}-T^{19}$ & $\begin{array}{l}\text { GGGAAAGCUAAGGACUGGUUGCUGCAGGCUGCUUGUACCAAUUGCUAUACUCGAGAAUAGCAAUUGGUAC } \\
\text { AAGCAGGGACGUCGAACCAGUCCUUAGCUUUCCUx }\end{array}$ & 106 \\
\hline
\end{tabular}

a: sequences external to the target-matched dsRNA domains are shown in bold (e.g. loops, spacers, $U_{x}$ termination remnants).

suppressive activity of each hairpin was progressively reduced with increasing numbers of hairpins coexpressed. This was equally applicable for all three hairpin co-expression strategies; multiple vectors, multiple cassettes, and multiple domains. Hairpin competition was evident in all cases except one, where the activity of the Vpu 158-29 hairpin was little affected when transiently expressed with 3 other hairpins from a 4 cassette vector. Although the reasons for this are unknown to us, we note that this hairpin was very active and so it may be that even as it competed with 3 other shRNA its suppressive impact was unaffected. Overall, we surmise that hairpins interact competitively for access to the RNAi machinery. Whilst this conclusion is supported by some [45-47], it is worth noting that there are also conflicting conclusions, where others report no evidence of shRNA/ siRNA competition $[29,30,48,49]$. The reason for this disparity may relate to differences in experimental design such as expression levels and observations under subsaturating conditions. It should also be noted that issues of shRNA competition in mammalian cells encompass endogenous RNAi substrates as well, e.g. microRNA. Expression levels in a clinical setting may need to be finely tuned to attain sufficient activity, with minimal impact [2,50-53]. Another idea for removing competition may be to employ multiple agents of different modalities (e.g. RNAi, aptamers and ribozymes) so that no single pathway is overwhelmed $[54,55]$.

When each hairpin of different sequence was directed to a common target (i.e. the complete HIV-1 sequence rather than individual gene-fusions), we saw that the net suppressive activity was approximately equivalent to the average activity of the component hairpins. This suggests that hairpin diversity may be increased whilst maintaining overall suppressive activity. This could potentially be exploited for countering the emergence of viral escape mutants in-line with other studies [27] though it requires further work for verification. Moreover, we did not test the effect on net activity of using one or more hairpins which was poor, or completely inactive (as all our hairpins here were classed as highly active). Such a situation could conceivably arise in a clinical setting due to a virus developing a mutation in one of the target sites. We speculate that the net suppressive activity would be reduced, though our mathematical modeling of various infection scenarios indicates that some loss of shRNA efficacy can be tolerated without impacting on treatment success [21,22].

Our data shows that up to 4 repeats of the same shRNA can increase the net suppressive activity when transiently expressed at levels below that which results in maximal 


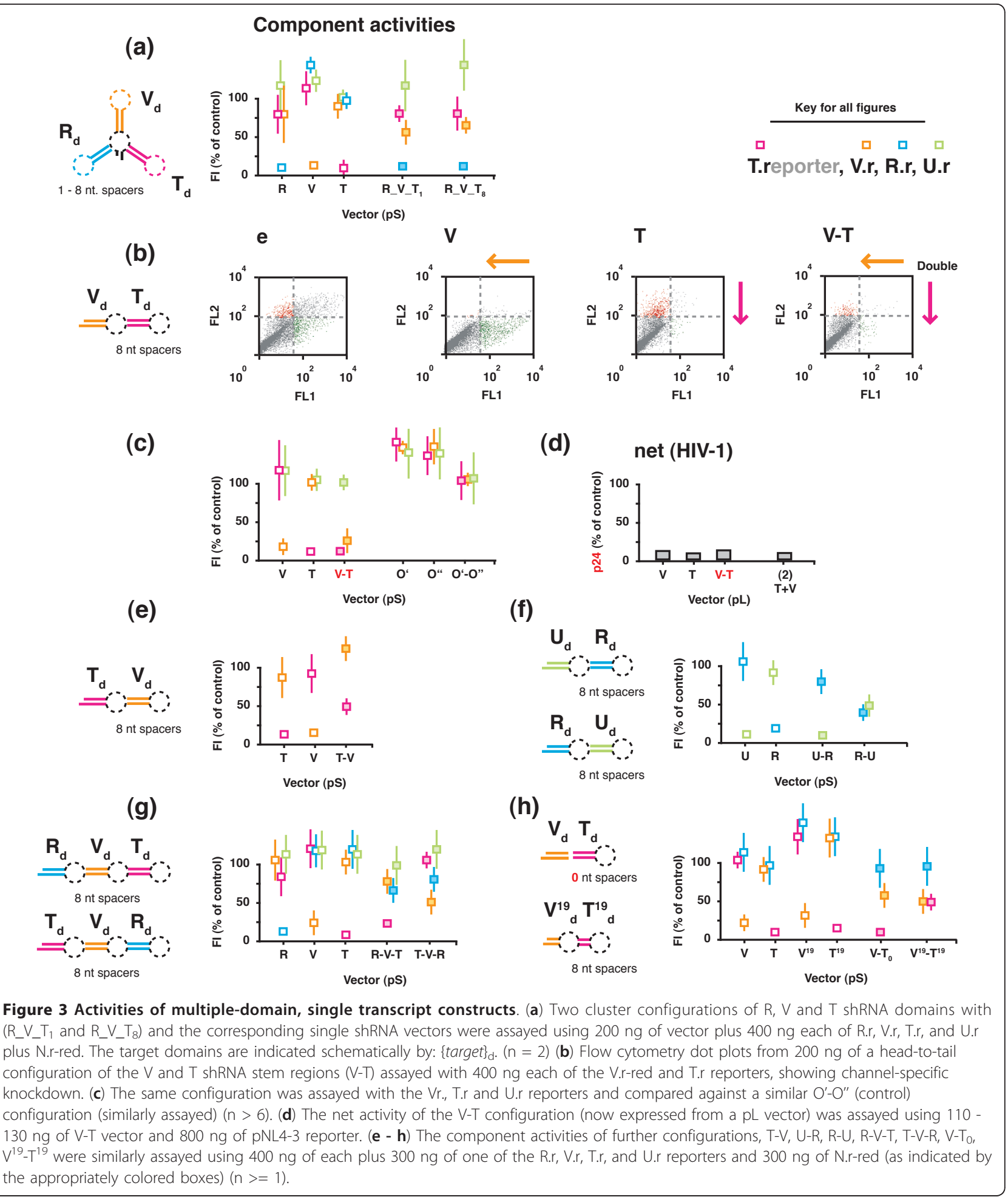

suppressive activity. Interestingly, the same effect was not seen in the corresponding stably transduced cell lines. The reasons for this are unclear, but could result from promoter interference [41], or transcriptional silencing [56]. Other studies have shown that promoter interference is not necessarily a barrier to multiple shRNA cassette strategies $[31,32,54]$. One study has shown that up to 6 identical expression cassettes could increase total expression and suppressive activity during both transient and stable expression, though in this case more than six cassettes was 
deleterious in stable expression as it decreased the net suppressive activity [32]. Repeat-mediated cassette deletion is also a concern, as we and others have since shown that it commonly occurs during infection [21,51], possibly via reverse transcriptase slipping on its template [57], though again, our modeling suggests that the practical impact of this in a gene therapy setting may be low [21]. Finally, the reduction in titre that we observed, whilst inconsequential here, has also been noted by others [42,58-60] and is an issue that may have to be addressed prior to scaled-up manufacturing.

Our attempts to generate a cluster of 3 shRNA domains in a single transcript with activity retained equally in each domain were unsuccessful. However, due to the highly structured templates, we were unable to confirm the sequence of these templates with automated sequencing procedures. Thus we cannot rule out the possibility of single nucleotide errors in these configurations. This is another issue that needs to be overcome, though Liu et. al. have reported success by incorporating several G:U wobbles [61]. Head-to-tail configurations can be sequenced though (using a modified protocol [62]), and in this respect may be a more attractive choice. However, since these are akin to 'long' hairpins, they in turn may induce non-specific dsRNAresponse pathways such as protein kinase $R$ (PKR) or interferon (IFN) [63,64], though recent work suggests otherwise [61]. Even though our designs incorporated spacers (of 1 or $8 \mathrm{nt}$.) to keep all regions of paired double-stranded RNA less than 30 bp (the minimal length traditionally thought to activate non-specific pathways), there are reports that some structures outside of this traditional view (e.g. $<30 \mathrm{bp}$ ) may also stimulate these responses [65-68]. At this point though, the principle limitation of these configurations is in not knowing how they are processed, and consequently how they should be designed to reliably retain activity in all domains. Based on our understanding of single shRNA processing, where a single nt. shift in the start of the shRNA stem can significantly alter activity, we speculate that the processed products from our multiple domain constructs are simply different from the those liberated from the 'corresponding' single shRNAs. Liu et. al. and others are making inroads in this area $[61,69]$, which will be well complemented by future deep-sequencing type studies. It is interesting to note that others who have also looked at similar structures have been similarly unable to produce effective silencing from more than 3 domains [61]. As a workaround one group has stacked several 2-domain structures, which could then be further used in combination with a multiple cassette type arrangement to increase targeting capacity [39].

In summary, we found that while all 3 co-expression strategies tested were effective, the multiple cassette strategy is a most useful method for immediate use in gene therapy. This is because it was easy to design, assemble, and is directly compatible with pre-existing shRNA already selected for high activity. It is worth noting that a similar study was published during the preparation of this manuscript, with similar conclusions, thus strengthening the validity of our findings [70]. Furthermore, we have since applied the multiple cassette strategy in several additional studies, including the development of a repeating modular cloning method (tested with up to 11 shRNAs), the assembly of combinations of up to 7 shRNAs to target entire subtypes of HIV-1, and a large-scale study around repeat-mediated deletion of 1 or more cassettes [21,71].

\section{Methods}

\section{shRNA design and vector construction}

Each shRNA was designed so that the sense or upper strand of the shRNA stem was homologous to the target (designed to give rise to the siRNA passenger strand) and the anti-sense or lower strand of the shRNA stem was complementary to the target (designed to give rise to the siRNA guide strand) (Table 1). Sense and antisense strands were connected by an 8 or 9 nt loop and all hairpins were expressed from a human $\mathrm{H} 1$ polymerase III (pol III) promoter with transcription presumably terminating at a run of 4 or more ' $\mathrm{T}$ ' residues in the included termination signal (TTTTTTGGA). Each shRNA insert was constructed using either annealed complementary oligonucleotides (oligos) or primer extension [62] to create a synthetic DNA insert that was cloned into a pSilencer 3.0-H1 derived vector (Ambion). The pSilencer derivative was generated by replacing the bla gene (ampicillin ${ }^{r}$ ) for the neo gene (kanamycin ${ }^{r}$ $/ G 418^{r}$ ). Single cassette pLenti6 based vectors were created by sub-cloning entire shRNA expression cassettes from the pSilencer based vectors into a derivative of pLenti6/V5-D-TOPO (Invitrogen). The pLenti6 derivative was generated by exchanging the CMV promoter, V5 epitope and SV40 terminator region for a multiple clone site (MCS) to facilitate the unique insertion of 1 to 4 self-contained (i.e. consisting of promoter, hairpin and terminator) shRNA expression cassettes. Multiple cassette pLenti6 based vectors were created by PCR amplification of the desired cassette(s) from the corresponding pSilencer based vector(s), which were then progressively inserted into the appropriate pLenti6 based vector downstream of any previous cassettes with a gap of $\sim 130$ bp separating each cassette. pSilencer based vectors were propagated in GT116 E. coli cells (a cell line specifically developed for the replication of hairpin containing vectors, Invivogen) and pLenti6 based vectors were propagated in Stbl3 E. coli cells (manufacturer recommended cell line, Invitrogen). DNA was extracted (Hi-speed Maxi-prep Kit, Qiagen), quantitated 
in triplicate and was sequence confirmed either by standard protocols or a modified protocol where required to enable automated sequencing of hairpin expression vectors possessing reaction-inhibiting secondary structure [62] (excluding the multiple hairpin 'cluster' configurations as indicated in the text).

\section{Reporter vector construction}

The fluorescent protein-target fusion reporter vectors, were constructed using EGFP (from pd4-d4EGFP-N1, BD Biosciences), AsRed1 (from pAsRed1-C1, BD Biosciences) or AmCyan (from pAmCyan-C1, BD Biosciences) and HIV-1 sequences (variant NL4-3, accession \#AF324493). Each vector was designed to produce a single mRNA transcript comprising the fluorescent protein fused to a downstream HIV-1 gene sequence but separated by multiple stop codons to ensure that only the first domain would be translated (the fluorescent protein). The sizes of reporter vectors deviated by no more than 10\%. The reporters used here included: GFPsTat (T.r) and AsRed1sVif (V.r-red), GFPsVif (V.r), GFPsVpr (R.r) and GFPsVpu (U.r), and AsRed1sNef (N.r-red).

\section{Fluorescence based shRNA activity assay}

Human Embryonic Kidney cells (HEK293a, sourced from the American Type Culture Collection) were seeded at a density of $4-5 \times 10^{5}$ cells/well in 6 well plates in $2 \mathrm{ml}$ of Dulbecco's modified eagle medium plus $10 \%$ fetal bovine serum (DMEM-10). Cells were transfected 1 day later using $1 \mu \mathrm{g}$ of total DNA (comprised of different amounts of shRNA and/or 1 or more reporter vectors as indicated in each figure) with $4 \mu \mathrm{l}$ of Lipofectamine 2000 (Invitrogen) in OptiMEM (Invitrogen) to a total volume of $100 \mu \mathrm{l} /$ well. Cells were analyzed by flow cytometry 2 days later (using either a FACsort or FACsCalibur instrument, BD Bioscience). The suppressive activity of each shRNA was measured as a change in fluorescence of the reporter(s) (FL1 for 'green' proteins and FL2 for 'red' proteins). The Fluorescence Index (FI) of cells in each channel was calculated by multiplying the geo mean of fluorescence by the percentage of cells that were fluorescent (only those cells gated above background). The FI was expressed as a percentage of the FI of cells transfected only with the corresponding empty expression control vector that expresses no hairpin. Target-specific shRNA activities were normalized to account for non-specific effects measured using an additional 'green' or 'red' off-target reporter to which the shRNA bore no homology, except for cases where the simultaneous activities of 2 shRNAs where measured using a 'green' reporter for one shRNA, and a 'red' reporter for the other shRNA. Most assays included a 29 bp off-target control shRNA (O'), which displayed no meaningful suppressive activity against any reporter, and thus was omitted from the graphs for clarity.

\section{Lentivirus production and infection}

293FT cells (Invitrogen) were seeded at a density of $5 \times$ $10^{6}$ cells/plate $(100 \mathrm{~mm}$ plates; $10 \mathrm{ml}$ DMEM-10) and were transfected 1 day later using $12 \mu \mathrm{g}$ of total DNA (comprised of $3 \mu \mathrm{g}$ pLenti6 based hairpin vector and $9 \mu \mathrm{g}$ packaging vectors, Invitrogen) with $36 \mu$ of Lipofectamine 2000 in OptiMEM to a total volume of $8 \mathrm{ml} /$ plate. Viruscontaining medium (VCM) was harvested at 2 - 3 days post-transfection, cold spun at $3000 \mathrm{rpm}$ for $15 \mathrm{~min}$. and stored at $-80^{\circ} \mathrm{C}$. Viral titres were calculated using HEK293a cells seeded at $1 \times 10^{5}$ cells/well (6 well plates; $2 \mathrm{ml}$ DMEM-10) which were infected with serial dilutions of VCMs ranging from $10^{-1}$ to $10^{-6}$ supplemented with $6 \mu \mathrm{g} / \mathrm{ml}$ of polybrene (hexadimethrine bromide, Sigma). Selective medium (DMEM-10 plus $10 \mu \mathrm{g} / \mathrm{ml}$ Blasticidin, Invitrogen) was applied to infected cells 2 days later and maintained for 10 - 14 days prior to Giemsa staining (Merck) and quantification of colony numbers, with titres calculated as infectious viral particles (IVF)/ml of VCM. Stably transduced cell lines were generated using HEK293a cells seeded at a density of $4 \times 10^{5}$ cells/plate (6 well plates; 2 ml DMEM-10) which were infected 1 day later with $2 \mathrm{ml}$ of VCM with an average MOI of $\sim 0.4$. Selective medium (DMEM-10 plus $10 \mu \mathrm{g} / \mathrm{ml}$ Blasticidin) was applied to infected cells 4 days later and maintained for at least 14 days.

\section{HIV-1 production assay}

HEK293a cells were seeded at a density of $2 \times 10^{5}$ cells/ well (12 well plates; $1 \mathrm{ml}$ of DMEM-10). Cells were transfected 1 day later using 110 - 130 ng of hairpin expression vector (at equimolar amounts across transfections) and $800 \mathrm{ng}$ ( $3 \times$ molar amount of expression vector) of pNL4-3 reporter vector (expressing the 4-3 strain of HIV-1) with Lipofectamine 2000 at a ratio of $1: 4$ ( $\mu \mathrm{g}$ DNA: $\mu$ l Lipofectamine) in OptiMEM to a total volume of $200 \mu \mathrm{l} /$ well. Medium was replaced with an equal volume 1 day post-transfection and the cells were harvested a further 1 day later by centrifugation at $400 \mathrm{~g}$ for $10 \mathrm{~min}$. at room temperature. Samples were stored at $-20^{\circ} \mathrm{C}$ until assayed for p24 levels (a capsid protein required for HIV-1 virion production) via EnzymeLinked Immunosorbent Assay (ELISA) using the INNOTEST HIV antigen mAb kit (Innogenetics). The suppressive activity of each shRNA was measured as a reduction in, and expressed as percentage of, $\mathrm{p} 24$ production (measured as $\mathrm{pg} / \mathrm{ml}$ ) relative to $\mathrm{p} 24$ production from cells transfected with the corresponding empty expression control vector. 


\section{Statistical analysis}

Each sample was analyzed in triplicate with 95\% confidence intervals (CI) calculated using Microsoft Excel X. $P$ values were determined by analysis of variance (ANOVA, with a Bonferroni's multiple test comparison) using Prism 4.0a.

\section{Acknowledgements}

GJM was a recipient of the Australian Postgraduate Award (APA). The authors thank Dr. Tanya Applegate, Emeritus Prof. Donald Birkett, Dr. Mehnaaz Lomas, Dr. Toby Passioura, Dr. Yi-Hsin Yu, Dr. Laurent Rivory and others at Johnson and Johnson Research for assistance in preparing the manuscript. This work was funded by Johnson and Johnson Research. Figures were prepared by http://www.madebyglen.com

\section{Author details}

${ }^{1}$ Johnson and Johnson Research Pty Ltd, Level 4 Biomedical Building, 1 Central Avenue, Australian Technology Park, Eveleigh, NSW, 1430, Australia. ${ }^{2}$ School of Biotechnology and Biomolecular Sciences, The University of New South Wales, Sydney, NSW 2052, Australia. ${ }^{3}$ Tibotec BVBA, Gen De Wittelaan L 11 B3, 2800 Mechelen, Belgium.

\section{Authors' contributions}

GJM and GCF conceived the experiments. GJM designed the constructs, made the plasmids and performed the fluorescence experiments. AJA, KMG, and WMM performed the Lentiviral production and HIV expression experiments. GJM wrote the manuscript. All authors have read and approved the final manuscript.

\section{Competing interests}

This work was done jointly by GJM, at the time a student enrolled in The school of Biotechnology and Biomedical Science at the University of New South Wales, Sydney, Australia, and employees of Johnson and Johnson Research (JJR), for Johnson and Johnson Research.

Received: 9 September 2010 Accepted: 17 April 2011

Published: 17 April 2011

\section{References}

1. Bartel D: MicroRNAs: genomics, biogenesis, mechanism, and function. Cell 2004, 116:281-297.

2. Kim V: MicroRNA biogenesis: coordinated cropping and dicing. Nat ReV Mol Cell Biol 2005.

3. Schwarz D, Hutvagner G, Du T, Xu Z, Aronin N, Zamore P: Asymmetry in the assembly of the RNAi enzyme complex. Cell 2003, 115:199-208.

4. Elbashir S, Harborth J, Lendeckel W, Yalcin A, Weber K, Tuschl T: Duplexes of 21-nucleotide RNAs mediate RNA interference in cultured mammalian cells. Nature 2001, 411:494-498.

5. Caplen N, Parrish S, Imani F, Fire A, Morgan R: Specific inhibition of gene expression by small double-stranded RNAs in invertebrate and vertebrate systems. Proc Natl Acad Sci USA 2001, 98:9742-9747.

6. Brummelkamp T, Bernards R, Agami R: A system for stable expression of short interfering RNAs in mammalian cells. Science 2002, 296:550-553.

7. Paddison P, Caudy A, Hannon G: Stable suppression of gene expression by RNAi in mammalian cells. Proc Natl Acad Sci USA 2002, 99:1443-1448.

8. Dickins R, Hemann M, Zilfou J, Simpson D, Ibarra I, Hannon G, Lowe S: Probing tumor phenotypes using stable and regulated synthetic microRNA precursors. Nat Genet 2005.

9. Lee M, Coburn G, McClure M, Cullen B: Inhibition of human immunodeficiency virus type 1 replication in primary macrophages by using Tat- or CCR5-specific small interfering RNAs expressed from a lentivirus vector. J Virol 2003, 77:11964-11972.

10. Rubinson D, Dillon C, Kwiatkowski A, Sievers C, Yang L, Kopinja J, Rooney D, Ihrig M, McManus M, Gertler F, et al: A lentivirus-based system to functionally silence genes in primary mammalian cells, stem cells and transgenic mice by RNA interference. Nat Genet 2003, 33:401-406.

11. ter Brake $\mathrm{O}$, Konstantinova P, Ceylan M, Berkhout B: Silencing of HIV-1 with RNA interference: a multiple shRNA approach. Mol Ther 2006, 14:883-892.
12. Mcintyre G, Groneman J, Yu Y, Jaramillo A, Shen S, Applegate T: 96 shRNAs designed for maximal coverage of HIV-1 variants. Retrovirology 2009, 6:55.

13. Naito Y, Nohtomi K, Onogi T, Uenishi R, Ui-Tei K, Saigo K, Takebe Y: Optimal design and validation of antiviral siRNA for targeting HIV-1. Retrovirology 2007, 4:80.

14. Das AT, Brummelkamp TR, Vink M, Madiredjo M, Bernards R, Berkhout B: Human immunodeficiency virus type 1 escapes from RNA interferencemediated inhibition. Journal of Virology 2004, 78:2601-2605.

15. Boden, Pusch, Lee, Tucker, Ramratnam : Human immunodeficiency virus type 1 escape from RNA interference. Journal of Virology 2003, 77:11531-11535

16. Westerhout EM, Ooms M, Vink M, Das AT, Berkhout B: HIV-1 can escape from RNA interference by evolving an alternative structure in its RNA genome. Nucleic Acids Res 2005, 33:796-804.

17. Nishitsuji : Effective Suppression of Human Immunodeficiency Virus Type 1 through a Combination of Short- or Long-Hairpin RNAs Targeting Essential Sequences for Retroviral Integration. Journal of Virology 2006 80:7658-7666.

18. Ter brake O, Berkhout B: A novel approach for inhibition of HIV-1 by RNA interference: counteracting viral escape with a second generation of siRNAs. Journal of RNAi and Gene Silencing 2005, 1:56-65.

19. Wilson JA, Richardson CD: Hepatitis $C$ virus replicons escape RNA interference induced by a short interfering RNA directed against the NS5b coding region. J Virol 2005, 79:7050-7058.

20. Leonard JN, Schaffer DV: Computational design of antiviral RNA interference strategies that resist human immunodeficiency virus escape. J Virol 2005, 79:1645-1654.

21. Mclntyre $G, Y u Y$, Tran A, Jaramillo A, Arndt A, Millington M, Boyd M, Elliott F, Shen S, Murray J, Applegate T: Cassette deletion in multiple shRNA lentiviral vectors for HIV-1 and its impact on treatment success. Virol J 2009, 6:184.

22. Applegate $T L$, Birkett DJ, McIntyre GJ, Jaramillo AB, Symonds G, Murray JM In silico modeling indicates the development of HIV-1 resistance to multiple shRNA gene therapy differs to standard antiretroviral therapy. Retrovirology 2010, 7:83.

23. Nishitsuji $\mathrm{H}$ : Effective Suppression of Human Immunodeficiency Virus Type 1 through a Combination of Short- or Long-Hairpin RNAs Targeting Essential Sequences for Retroviral Integration. Journal of Virology 2006, 80:7658-7666.

24. ter Brake O, 't Hooft K, Liu YP, Centlivre M, von Eije KJ, Berkhout B: Lentiviral vector design for multiple shRNA expression and durable HIV1 inhibition. Mol Ther 2008, 16:557-564.

25. Liu YP, Haasnoot J, Ter Brake O, Berkhout B, Konstantinova P: Inhibition of HIV-1 by multiple siRNAs expressed from a single microRNA polycistron. Nucleic Acids Research 2008, 36:2811-2824.

26. Von Eije KJ, Brake OT, Berkhout B: Human Immunodeficiency Virus Type 1 Escape Is Restricted When Conserved Genome Sequences Are Targeted by RNA Interference. Journal of Virology 2008, 82:2895-2903.

27. Schopman NCT, Ter Brake O, Berkhout B: Anticipating and blocking HIV-1 escape by second generation antiviral shRNAs. Retrovirology 2010, 7:52.

28. Méndez-Ortega MC, Restrepo S, Rodríguez-R LM, Pérez I, Mendoza JC, Martínez AP, Sierra R, Rey-Benito GJ: An RNAi in silico approach to find an optimal shRNA cocktail against HIV-1. Virology Journal 2010, 7:369.

29. Yu J, Taylor J, DeRuiter S, Vojtek A, Turner D: Simultaneous inhibition of GSK3alpha and GSK3beta using hairpin siRNA expression vectors. Mol Ther 2003, 7:228-236.

30. Schuck S, Manninen A, Honsho M, Fullekrug J, Simons K: Generation of single and double knockdowns in polarized epithelial cells by retrovirusmediated RNA interference. Proc Natl Acad Sci USA 2004, 101:4912-4917.

31. Anderson J, Akkina R: HIV-1 resistance conferred by siRNA cosuppression of CXCR4 and CCR5 coreceptors by a bispecific lentiviral vector. AIDS Res Ther 2005, 2:1.

32. Gonzalez S, Castanotto D, Li H, Olivares S, Jensen M, Forman S, Rossi J, Cooper L: Amplification of RNAi-targeting HLA mRNAs. Mol Ther 2005, 11:811-818.

33. Gou D, Weng T, Wang Y, Wang Z, Zhang H, Gao L, Chen Z, Wang P, Liu L: A novel approach for the construction of multiple shRNA expression vectors. J Gene Med 2007, 9:751-763.

34. Henry S, Vanderwegen $\mathrm{P}$, Metselaar $\mathrm{H}$, Tilanus $\mathrm{H}$, Scholte B, Vanderlaan L: Simultaneous targeting of HCV replication and viral binding with a 
single lentiviral vector containing multiple RNA interference expression cassettes. Mol Ther 2006, 14:485-493.

35. Saayman, Barichievy, Capovilla, Morris, Arbuthnot, Weinberg, Bowyer: The Efficacy of Generating Three Independent Anti-HIV-1 siRNAs from a Single U6 RNA Pol III-Expressed Long Hairpin RNA. PLOS ONE 2008, 3: e2602.

36. Liu, Haasnoot, Berkhout: Design of extended short hairpin RNAs for HIV-1 inhibition. Nucleic Acids Research 2007, 35:5683-5693.

37. Sano , Li , Nakanishi, Rossi : Expression of Long Anti-HIV-1 Hairpin RNAs for the Generation of Multiple siRNAs: Advantages and Limitations. Mo Ther 2008, 16:170-177.

38. Zhu X, Santat LA, Chang MS, Liu J, Zavzavadjian JR, Wall EA, Kivork C, Simon Ml, Fraser ID: A versatile approach to multiple gene RNA interference using microRNA-based short hairpin RNAs. BMC Mol Biol 2007, 8:98.

39. Saayman S, Arbuthnot P, Weinberg MS: Deriving four functional anti-HIV siRNAs from a single Pol III-generated transcript comprising two adjacent long hairpin RNA precursors. Nucleic acids research 2010, 38:6652.

40. Mcintyre G: Antiviral shRNA (for HIV-1). Biotechnology \& Biomolecular Sciences, Faculty of Science, UNSW 2006, 1-343.

41. Cullen B: Induction of stable RNA interference in mammalian cells. Gene Ther 2005.

42. Miyagishi M, Sumimoto H, Miyoshi H, Kawakami Y, Taira K: Optimization of an siRNA-expression system with an improved hairpin and its significant suppressive effects in mammalian cells. J Gene Med 2004, 6:715-723.

43. Lakka S, Gondi C, Yanamandra N, Olivero W, Dinh D, Gujrati M, Rao J: Inhibition of cathepsin B and MMP-9 gene expression in glioblastoma cell line via RNA interference reduces tumor cell invasion, tumor growth and angiogenesis. Oncogene 2004, 23:4681-4689.

44. Zeng $Y$, Cullen B: Sequence requirements for micro RNA processing and function in human cells. Rna 2003, 9:112-123.

45. McManus M, Haines B, Dillon C, Whitehurst C, Van Parijs L, Chen J, Sharp P: Small interfering RNA-mediated gene silencing in T lymphocytes. J Immunol 2002, 169:5754-5760

46. Holen T, Amarzguioui M, Wiiger M, Babaie E, Prydz H: Positional effects of short interfering RNAs targeting the human coagulation trigger Tissue Factor. Nucleic Acids Res 2002, 30:1757-1766.

47. Castanotto D, Sakurai K, Lingeman R, Li H, Shively L, Aagaard L, Soifer $H$, Gatignol A, Riggs A, Rossi Jj: Combinatorial delivery of small interfering RNAs reduces RNAi efficacy by selective incorporation into RISC. Nucleic Acids Res 2007, 35:5154-5164.

48. Kahana R, Kuznetzova L, Rogel A, Shemesh M, Hai D, Yadin H, Stram Y: Inhibition of foot-and-mouth disease virus replication by small interfering RNA. J Gen Virol 2004, 85:3213-3217.

49. Ji J, Wernli M, Klimkait T, Erb P: Enhanced gene silencing by the application of multiple specific small interfering RNAs. FEBS Lett 2003, 552:247-252.

50. Hannon G, Rossi J: Unlocking the potential of the human genome with RNA interference. Nature 2004, 431:371-378.

51. Leonard J, Schaffer D: Antiviral RNAi therapy: emerging approaches for hitting a moving target. Gene Ther 2005.

52. Huppi K, Martin S, Caplen N: Defining and Assaying RNAi in Mammalian Cells. Mol Cell 2005, 17:1-10.

53. Yi R, Doehle B, Qin Y, Macara I, Cullen B: Overexpression of exportin 5 enhances RNA interference mediated by short hairpin RNAs and microRNAs. Rna 2005, 11:220-226.

54. Li M, Kim J, Li S, Zaia J, Yee J, Anderson J, Akkina R, Rossi J: Long-Term Inhibition of HIV-1 Infection in Primary Hematopoietic Cells by Lentiviral Vector Delivery of a Triple Combination of Anti-HIV shRNA, Anti-CCR5 Ribozyme, and a Nucleolar-Localizing TAR Decoy. Mol Ther 2005.

55. Banerjea A, Li M, Remling L, Rossi J, Akkina R: Lentiviral transduction of Tar Decoy and CCR5 ribozyme into CD34+ progenitor cells and derivation of HIV-1 resistant T cells and macrophages. AIDS Res Ther 2004, 1:2.

56. Morris K, Rossi J: Lentiviral-mediated delivery of siRNAs for antiviral therapy. Gene Ther 2006, 13:553-558.

57. Brake O, Hooft K, Liu Y, Centlivre M, Jasmijn von Eije K, Berkhout B: Lentiviral Vector Design for Multiple shRNA Expression and Durable HIV1 Inhibition. Mol Ther 2008, 16:557-564.

58. Chang L, Liu X, He J: Lentiviral siRNAs targeting multiple highly conserved RNA sequences of human immunodeficiency virus type 1 . Gene Ther 2005.
59. Abbas-Terki T, Blanco-Bose W, Deglon N, Pralong W, Aebischer P: Lentiviralmediated RNA interference. Hum Gene Ther 2002, 13:2197-2201.

60. Poluri A, Sutton RE: Titers of HIV-based Vectors Encoding shRNAs are Reduced by a Dicer-dependent Mechanism. Mol Ther 2008, 16:378-386.

61. Liu YP, Eije KJV, Schopman NC, Westerink J-T, Brake Ot, Haasnoot J, Berkhout B: Combinatorial RNAi Against HIV-1 Using Extended Short Hairpin RNAs. Molecular Therapy 2010, 17:1712-1723.

62. Mclntyre GJ, Fanning GC: Design and cloning strategies for constructing shRNA expression vectors. BMC Biotechnol 2006, 6:1.

63. Samuel C: Antiviral actions of interferons. Clin Microbiol Rev 2001, 14:778-809, table of contents.

64. Manche L, Green S, Schmedt C, Mathews M: Interactions between doublestranded RNA regulators and the protein kinase DAl. Mol Cell Biol 1992, 12:5238-5248.

65. Bridge A, Pebernard S, Ducraux A, Nicoulaz A, Iggo R: Induction of an interferon response by RNAi vectors in mammalian cells. Nat Genet 2003, 34:263-264.

66. Pebernard $\mathrm{S}$, lggo R: Determinants of interferon-stimulated gene induction by RNAi vectors. Differentiation 2004, 72:103-111.

67. Scacheri P, Rozenblatt-Rosen O, Caplen N, Wolfsberg T, Umayam L, Lee J, Hughes C, Shanmugam K, Bhattacharjee A, Meyerson M, Collins F: Short interfering RNAs can induce unexpected and divergent changes in the levels of untargeted proteins in mammalian cells. Proc Natl Acad Sci USA 2004, 101:1892-1897.

68. Samuel C: Knockdown by RNAi-proceed with caution. Nat Biotechnol 2004, 22:280-282.

69. Liu YP, Haasnoot J, Berkhout B: Design of extended short hairpin RNAs for HIV-1 inhibition. Nucleic Acids Research 2007, 35:5683-5693.

70. Lambeth LS, Van Hateren NJ, Wilson SA, Nair V: A direct comparison of strategies for combinatorial RNA interference. BMC Mol Biol 2010, 11:77.

71. Mcintyre GJ, Groneman JL, Tran A, Applegate TL: An Infinitely Expandable Cloning Strategy plus Repeat-Proof PCR for Working with Multiple ShRNA. PLOS ONE 2008, 3:e3827.

doi:10.1186/1479-0556-9-9

Cite this article as: Mcintyre et al: A comparison of multiple shRNA expression methods for combinatorial RNAi. Genetic Vaccines and Therapy 2011 9:9.

\section{Submit your next manuscript to BioMed Central and take full advantage of:}

- Convenient online submission

- Thorough peer review

- No space constraints or color figure charges

- Immediate publication on acceptance

- Inclusion in PubMed, CAS, Scopus and Google Scholar

- Research which is freely available for redistribution

Submit your manuscript at www.biomedcentral.com/submit
C Biomed Central 